\title{
1 A powerful framework for an integrative study with heterogeneous omics data: from
}

\section{2 univariate statistics to multi-block analysis}

4 Harold Duruflé ${ }^{1,2}$, Merwann Selmani ${ }^{1}$, Philippe Ranocha ${ }^{1}$, Elisabeth Jamet $^{1}$, Christophe

5 Dunand $^{1 *}$, Sébastien Déjean ${ }^{3 *}$

$7 \quad{ }^{1}$ Laboratoire de Recherche en Sciences Végétales, Université de Toulouse, CNRS, UPS, 24

8 chemin de Borde Rouge, Auzeville, BP 42617, 31326 Castanet-Tolosan, France.

$9 \quad{ }^{2}$ LIPM, Université de Toulouse, INRA, CNRS, Castanet-Tolosan, France.

$10{ }^{3}$ Institut de Mathématiques de Toulouse, Université de Toulouse, CNRS, UPS, 31062

11 Toulouse, France

$12{ }^{*}$ Corresponding authors:

13 Sébastien Déjean (sebastien.dejean@math.univ-toulouse.fr; +33 (0)5 615569 16)

14 Christophe Dunand (dunand@lrsv.ups-tlse.fr; +33 (0)5 343238 58) 


\section{ABSTRACT}

18 The high-throughput data generated by new biotechnologies used in biological studies require

19 specific and adapted statistical treatments. In this work, we propose a novel and powerful

20 framework to manage and analyse multi-omics heterogeneous data to carry out an integrative

21 analysis. We illustrate it using the package mixOmics for the R software as it specifically

22 addresses data integration issues. Our work also aims at confronting the most recent

23 functionalities of mixOmics to real data sets because, even if multi-block integrative

24 methodologies exist, they still have to be used to enlarge our know-how and to provide an

25 operational framework to biologists. Natural populations of the model plant Arabidopsis

26 thaliana are employed in this work but the framework proposed is not limited to this plant and

27 can be deployed whatever the organisms of interest and the biological question. Four omics

28 data sets (phenomics, metabolomics, cell wall proteomics and transcriptomics) have been

29 collected, analysed and integrated in order to study the cell wall plasticity of plants exposed to

30 sub-optimal temperature growth conditions. The methodologies presented start from basic

31 univariate statistics and lead to multi-block integration analysis, and we highlight the fact that

32 each method is associated to one biological issue. Using this powerful framework led us to

33 novel biological conclusions that could not have been reached using standard statistical

34 approaches.

36 Keywords: abiotic stress, Arabidopsis thaliana, integrative analysis, statistical framework,

37 systems biology. 


\section{INTRODUCTION}

Biological processes can be studied using measurements that are ever more complex.

40 Today, biologists have access to plethora of new technologies to address their questions. The

41 high-throughput measurements have revolutionized the way to evaluate and predict the

42 behavior of organisms for example in response to environmental changes. Nowadays, one

43 biological sample can deliver many types of "big" data, such as genome sequences

44 (genomics), genes and proteins expression levels (transcriptomics and proteomics), metabolite

45 profiles (metabolomics) and phenotypic observations (phenomics). The revolution of high

46 throughput technologies has also greatly reduced the cost of those omics data production,

47 opening new prospects to the development of tools for data treatment and analysis ( $\mathrm{Li}, \mathrm{Wu}, \&$

48 Ngom, 2016; Meng et al., 2016).

The heterogeneous data collected from cellular to organism levels are associated to a

50 wide variety of techniques sometimes species-specific. The acquisition of data requires a

51 particular experimental design and a suitable methodology to highlight their mining (Rai,

52 Saito, \& Yamazaki, 2017). An experimental design inadequate for an integrative analysis

53 could complicate the final interpretation of the collected data. On the contrary, a suitable

54 methodology of analysis can be optimized and brings keys to improve the visibility of the

55 whole data. This point of view was previously stated in (Kerr, 2003) for microarray studies:

56 "While a good design does not guarantee a successful experiment, a suitably bad design

57 guarantees a failed experiment—no results or incorrect results”.

58 Use of multi-omics data makes possible a deeper understanding of a biological system

59 (Zargar et al., 2016, Rajasundaram \& Selbig, 2016). Indeed, quantification technologies

60 improve accuracy and create great potential for elucidating new questions in biology.

61 However, this technological revolution must be carefully used because the correlation

62 between quantification analyses is not effective. For example, it is known that it is usually 
63 difficult to correlate transcriptomic and proteomic data (Duruflé et al., 2017; Jamet et al.,

64 2009; Maier, Güell, \& Serrano, 2009). Each of these technologies has its own limitations and

65 collecting different types of data should help understanding the effects of one or more

66 experimental conditions. A cohort of hypotheses can be proposed with multi-omics analysis.

67 Thus, biological candidates can be identified as biomarkers (e.g. genes, proteins, molecules)

68 under complex environmental conditions, and/or new complex regulations can be found.

Altogether, it is generally admitted that studying a single kind of omics data is not

70 sufficient to understand the effects of a treatment on a complex biological system. To obtain a

71 holistic view, it is preferable to combine multiple omics analyses. To highlight the interest of

72 such integrative approaches, let us consider a toy example with two variables (Vx and Vy)

73 measured on 12 individuals (6 from one group called Controlled, and 6 from another one

74 called Treated). The values are presented in supplemental Table S1. Statistical tests

75 (Wilcoxon rank sum test and Student t test) do not reveal any significant difference between

76 the two groups for both the $\mathrm{Vx}$ and Vy variables when they are analysed separately (p-values

77 higher than 0.3). But, a simple scatterplot (Figure 1) highlights the interest of combining the

78 two variables. Indeed, it clearly appears that the two groups are separated if we consider the

$79 \quad \mathrm{Vx}$ and Vy variables together.

Thus, in the same vein, we claim that the integrative analysis of several data sets

81 acquired on the same individuals can reveal information that single data set analysis would

82 keep hidden. Furthermore, the toy example also highlights the interest of a relevant graphical

83 representation: information hidden in supplemental Table S1 is clearly visible in Figure 1.

84 The recent work by (Matejka \& Fitzmaurice, 2017) is assuredly a good way to be strongly

85 convinced about data visualisation.

This article focuses on a powerful framework we propose to manage and analyse

87 heterogeneous data sets acquired on the same samples. It proceeds step by step, from basic 
88 univariate statistics to multi-block integration analysis (Singh et al., 2016; Tenenhaus et al.,

89 2014). We illustrate the gaps bridged by each method from the computation of univariate

90 statistics to a thorough implementation of multi-block exploratory analysis. The

91 implementation of the methods and the graphical visualizations have simply been

92 accomplished with existing tutorials for the R software (R Core Team, 2018) and the

93 mixOmics package (Rohart et al., 2017). But, since their interpretation is not easy (González

94 et al., 2013), this article will provide a better understanding of the statistical integration and a

95 way to include it in a global reflexion structured in a workflow summarized in Figure 2. We

96 also aim at increasing our know-how related to these novel methodologies by confronting

97 them to new real data sets. The first section presents the background of our study and the data

98 sets we have dealt with detailed in (Duruflé, 2019a; 2019b). Then, we describe several

99 statistical methods used to address specific biological questions. Afterwards, we explain in

100 detail the statistical results and give clues to interpret them.

\section{BIOLOGICAL CONTEXT}

In the global warming context, seasons are altered with modifications of the

104 temperatures. The elevation of the temperature is the most studied change because it is 105 already observed (Savo et al., 2016). The occurrence of cold stress can also appear without 106 any previous chilling period and it could become a problem to maintain agricultural 107 productivity in the future (Gray \& Brady, 2016). The model plant Arabidopsis thaliana of the

108 Brassicaceae family has a worldwide geographical distribution and therefore has to adapt to 109 multiple and contrasted environmental conditions (Hoffmann, 2002). The huge accumulation 110 of molecular data concerning this plant is very helpful for studying complex multiple levels 111 responses. It is expected to transfer obtained results to other plant species of economic interest 112 for translational pipelines (Sibout, 2017). 
114 2.1. Experimental design

First, a compromise is necessary to determine the ideal number of biological

116 replicates. It is hard to find an agreement between the reality of the biological experimentation

117 (e.g. limitation in material, space, time, work force and cost) and the necessity to get robust

118 information for the statistical analyses. The method used for the randomization of the

119 replicate also needs to be considered. For these reasons, the experimental protocol must 120 minimize potential external impacts within and between the replicates and avoid confounded 121 effects.

To strengthen the results, each biological replicate can be the average of several

123 technical replicates, if the type of analysis allows it. For the biologist, it is important to know

124 the number of experimental repetitions to appreciate the variability between the different

125 conditions. But, for a statistician, the information resides into the intrinsic variability of the 126 different samples or repetitions. For all these reasons, one sample considered as "out of 127 norms” by the biologist could be valuable in a multi-omics analysis.

129 (4 Pyrenees Mountain ecotypes Roch, Grip, Hern, Hosp, living at different altitudes, and Col,

130 a reference ecotype from Poland, living at low altitude) and ii) temperature with 2 levels

$131\left(22^{\circ} \mathrm{C}\right.$ and $\left.15^{\circ} \mathrm{C}\right)$. For each ecotype, rosettes and floral stems were collected and analysed. At

$13222^{\circ} \mathrm{C}$, rosettes were collected at 4 weeks, i.e. at the time of floral stem emergence. At $22^{\circ} \mathrm{C}$,

133 floral stems were collected at 6, 7 and 8 weeks respectively for Col, Roch / Grip and Hern /

134 Hosp. At $15^{\circ} \mathrm{C}$, rosettes and stems were collected 2 weeks later than at $22^{\circ} \mathrm{C}$. More details

135 about the plant culture conditions can be found in (Duruflé, 2019a). Three independent

136 biological replicates were analysed for each sample including 20 plants per sample. To 
137 minimize the experimental effect, each plant was grown at a randomly chosen place according

138 to the experimental design represented in Figure 3.

141 In this project, the four following omics data sets (called blocks thereafter) were collected:

142 (i) Phenomics, i.e. a macro phenotyping analysis, was performed on two organs: rosettes and floral stems (Duruflé, 2019a). Indeed at the time of sample collection and prior to

(ii) Metabolomics, i.e. identification and quantification of seven cell wall freezing, 9 phenotypic variables were measured: 5 on rosettes (mass, diameter, number of leaves, density, and projected rosette area), and 4 on floral stems (mass, diameter, number of cauline leaves, length). monosaccharides (fucose, rhamnose, arabinose, galactose, glucose, xylose and galacturonic acid), were performed as previously described (Duruflé et al., 2017). Theoretical cell wall polysaccharide composition was inferred, based on the monosaccharide analyses according to (Duruflé et al., 2017; Houben et al. 2011; Duruflé, 2019a).

(iii) Proteomics, i.e. identification and quantification of cell wall proteins by LC-MS/MS analyses, were performed as described (Duruflé et al., 2017). Altogether, 364 and 414 cell wall proteins (CWPs) were identified and quantified in rosettes and floral stems, respectively (Duruflé, 2019b).

(iv) Transcriptomics, i.e. sequencing of transcripts also called RNA-seq, was performed according to the standard Illumina protocols as described (Duruflé et al., 2017). Altogether, 19763 and 22570 transcripts were analysed in rosettes and floral stems, respectively (Duruflé, 2019b). 


\section{TIDYING DATA}

Statistical data analysis requires efficient data pre-processing. As mentioned in

164 (Wickham, 2014), "It is often said that $80 \%$ of data analysis is spent on the process of cleaning and preparing the data". So in an integrative analysis framework, each data set needs to be structured in the same way, and (Wickham, 2014) has also stressed the following statements: 1/ Each variable forms a column. 2/ Each observation forms a row. So, in our context, each data set is structured with biological samples in rows and variables in columns. American statistician of the $20^{\text {th }}$ century), "the best thing to do with missing values is not to

171 have any”. Fortunately, many methods exist to deal with missing values. For instance, the 172 methodologies implemented in the missMDA package (Husson \& Josse, 2013) are dedicated 173 to the handling of missing values in the context of multivariate data analysis. For example in 174 this work, missing proteomics quantification data were dealt with considering two situations: 175 (i) non-validated proteins (identification with a single specific peptide and/or in a single 176 biological replicate); and (ii) undetectable proteins (no peptide identified in a given 177 condition). In the former case, a background noise, corresponding to the minimum, and the 178 first statistical quartile of the biological replicate, was applied. In the latter case, a background 179 noise of 6 (value lower than the minimum value found in the whole experiment) was applied. 180 This treatment allowed combining the quantification process with the qualitative study and 181 provided a higher confidence in the final result. More recently, a study focused on missing rows in data sets in an integrative 183 framework (Voillet et al., 2016). Within an integrative study, we can easily be in this case if, 184 for instance, the number of biological replicates is not the same for transcriptomics and 185 proteomics analyses. The main idea to remember would be to deal with missing values with 
an ad-hoc method taking into account the specificity of the data. In our case, two replicates of the transcriptomic data had to be deleted due to their low quality. Following the method proposed in (Voillet et al., 2016), these missing rows were imputed using the samples for which all the data were available, i.e. the two other replicates.

\section{RATIONALE SUPPORTING THE PROPOSED FRAMEWORK}

4.1. Software

As mentioned in the Comprehensive R Archive Network (CRAN, cran.r-project.org), $R$ "is a freely available language and environment for statistical computing and graphics which provides a wide variety of statistical and graphical techniques: linear and nonlinear modelling, statistical tests, time series analysis, classification, clustering, etc.”

R functions with a command-line interface that, even if it can appear not user-friendly, allows the user to build scripts that can be run on various data sets with rather few tuning. $\mathrm{R}$ gives access to the newest methodological developments due to its very active community (Rbloggers, R-help, UseR conference...) motivated by open science considerations. Furthermore, efficient tools such as RStudio (www.rstudio.org) were developed in order to make the initiation to $\mathrm{R}$ easier. In addition, many resources are available on CRAN to start with R. Therefore, it seems highly reasonable to expect that the user can read, use and adapt existing scripts available in the examples of each manual of packages after few hours of practice. Specifically considering the community of biologists using R, the Bioconductor repository (http://www.rstudio.org/) provides selected tools for the analysis of high-throughput genomic data (Gentleman et al., 2004).

The dynamism around $\mathrm{R}$ appears in the packages developed by and for the community. So, several packages exist to address statistical integrative studies. We focus on 
210 the mixOmics package (Lê Cao et al., 2009; Rohart et al., 2017), but other packages such as

211 FactoMineR (Husson \& Josse, 2013) can also be used for nearly similar purposes.

212 Methodologies presented in (Bécue-Bertaut \& Pagès, 2008) and (Sabatier et al., 2013) are

213 also alternatives, as well as the Multi-Omics Factor Analysis (MOFA) approach proposed in

214 (Argelaguet et al., 2018). Regarding commercial software for instance, SIMCA-P (Umetrics,

215 umetrics.com/) propose several methods to perform integrative analyses, and toolboxes for

216 Matlab are also available (The MathWorks, Inc., Natick, Massachusetts, United States). We

217 choose to favor an open source software, as it is easier to promote a free software than a

218 commercial one when people are not specialists in the domain (Carey \& Papin, 2018).

219 Furthermore, mixOmics appears as a very active package addressing data integration issues. It

220 has been downloaded more than 25,000 times (unique IP address) in 2017, 5 versions were

221 released in 2017, the reference article (Lê Cao et al., 2009) has been cited 300 times and the

222 mixOmics team has published 16 articles related to this package since 2008.

In this section, partly inspired from the tutorial of mixOmics (mixomics.org), we wish to

226 highlight the link between a biological question (purpose) and the appropriate statistical 227 method.

- Purpose: explore one single quantitative variable (e.g. what is the level of expression of one gene?). Method: univariate elementary statistics such as mean, median for main trends, and standard deviation or variance for dispersion, can be completed with a graphical representation such as boxplot. variable (e.g. Are the plant growth different in two or more environmental 
conditions?). Method: statistical significance test such as Student t test or Wilcoxon rank sum test for two groups and ANOVA or Kruskall-Wallis for more groups will address this question (McDonald, 2009). In this context, a special attention must be paid to the structure of the data: independent samples (e.g. independent groups observed in various conditions) or paired samples (e.g. same samples observed twice or more in various conditions). correlation between the concentration of one protein and its transcript abundance?). Method: correlation coefficients (Pearson for linear relationships and Spearman for monotonous ones) (McDonald, 2009). Graphical representations of correlation matrices can provide a global overview of pairwise indicators (Friendly, 2002; Murdoch \& Chow, 1996). patterns in the data, experimental bias or, identify if the samples 'naturally' cluster according to the biological conditions (e.g. Can we observe the effect of different environmental growth conditions on different ecotypes?). Method: an unsupervised variance, before performing PCA is usually useful when dealing with omics data to make the PCA results meaningful. factorial analysis such as Principal Component Analysis (PCA) (Mardia, Kent, \& Bibby, 1980) provides such information about one data set without any a priori on the result. Centering and scaling the data, such that all variables have zero mean and unit

257 data analysis whereas the methods mentioned hereafter are less usual. 

we classify various ecotypes according to their transcriptomics profile?). Method: supervised classification methods such as Partial Least Square Discriminant Analysis (PLS-DA) (Lê Cao, Boitard, \& Besse, 2011) assess how informative the data are to rightly classify samples, as well as to predict the class of new samples.

- Purpose: unravel the information contained in two data sets, where two types of variables are measured on the same samples (e.g. What are the main relationships between the proteomics and transcriptomics datasets?). Method: using PLS-related methods (Wold et al. , 2001) enable knowing if common information can be extracted from the two data sets (or highlight the relations between the two data sets).

The following methods are very recent and few applications have been published so far. This work contributes to improve their efficiency on real data sets.

- Purpose: the same as above but considering more than two data sets (e.g. What are the main relationships between the proteomics, transcriptomics and phenotypic data?). Method: multi-block PLS related methods were recently developed to address this issue (Günther et al., 2014; Singh et al., 2019).

- Purpose: the same as above but in a supervised context (e.g. Can we determine a multi-omics signature to classify ecotypes?). Method: multi-block PLS-DA (referred as DIABLO for Data Integration Analysis for Biomarker discovery using Latent variable approaches for Omics studies) was recently developed to address this issue (Singh et al., 2019). 
A schematic view of the data sets and the methods implemented is presented in Figure 4.

282 The way to perform an integrative statistical study is illustrated through several cycles (Figure

283

284

285 reduces the number of potentially relevant variables displayed on the graphical outputs. Thus, candidates for further investigations.

\subsection{Sparse extensions}

Every methods developed in mixOmics are proposed with a sparse extension (sparse PCA (S-PCA), sparse PLS (S-PLS)...). Sparse methods are useful to remove non-informative variables (e.g. which can be considered as background noise) regarding the purpose of the multivariate method. Concerning PCA for instance, the sparse version selects only the variables that highly contribute to the definition of each principal component (PC), removing the others. Sparsity is mathematically achieved via Least Absolute Shrinkage and Selection Operator (LASSO) penalizations (Tibshirani, 1996).

In practice, the use of sparse methods in the context of omics data is very useful as it it facilitates the biological interpretation of the results and minimizes the list of potential 
As previously mentioned, statistical analysis should be associated with graphical representations (Figure 4C). A famous sentence assigned to Francis John Anscombe (a British statistician of the $20^{\text {th }}$ century) emphasized this point of view: “... make both calculations and graphs. Both sorts of output should be studied; each will contribute to understanding."

309 (Anscombe, 1973). Based on this principle, a recent work by Matejka and Fitzmaurice

310 (Matejka \& Fitzmaurice, 2017) illustrates in a quite funny way how same numerical outputs can provide very different graphical representations (including a scatterplot looking like a dinosaur named datasaurus).

314 statistical testing. Boxplots and barplots, as produced, for instance, by the ggplot2 package 315 (Wickham, 2016), may complete and reinforce the interpretation of the results (Figure 4C).

316 Regarding barplots, one core question relies on the error bars that are frequently added: 317 should they be based on standard deviation or on standard error of the mean? A thorough 318 explanation about the difference is provided in (Cumming, Fidler, \& Vaux, 2007). The 319 authors also mention this statement that may seem obvious but that is sometimes forgotten: 320 "However, if $n$ is very small (for example $n=3$ ), rather than showing error bars and 321 statistics, it is better to simply plot the individual data points.” We also used graphical representations of correlation matrices (Figure 4C) such as 323 those produced by the corrplot package (Wei \& Simko, 2016) for the R software. This is 324 essential when dealing with (not so) many variables: with 50 variables, 1225 (50 x 49 / 2) 325 pairwise correlation coefficients are computed and have to be analysed and interpreted. Regarding multivariate analyses (from PCA to multi-block analyses), we used the 327 graphical outputs provided by the mixOmics R package (Rohart et al., 2017). They are based 328 on the representation of individuals and variables projected on specific sub-spaces (Figure 
329 4C). A thorough discussion about the complementarity between several graphical displays is 330 given in (González et al., 2013).

332 are represented as points located in a specific sub-space defined by the first PLS-components

333 (Figure 4C). Interpretation is based on the relative proximities of the samples and on the 334 equivalent representation for variables.

The standard representation for the variable plots is frequently referred as correlation 336 circle plot (Figure 4C). It was primarily used for PCA to visualise relationships between 337 variables, but it has been extended to deal with multi-block analysis. In such a plot, the 338 correlation between two variables can be visualised through the cosine of the angle between 339 two vectors starting at the origin and ending at the location of the point representing the 340 variable. The representation of variables can also be done through a relevance network. These 341 networks are inferred using a pairwise similarity matrix directly obtained from the outputs of 342 the integrative approaches (González et al., 2013). A Circos plot (Singh et al., 2019) can be

343 viewed as a generalization of relevance network where the nodes are located on a circle. 344 Then, based on the same pairwise similarity matrix used for relevance network, a clustered 345 image map can be displayed. This type of representation is based on a hierarchical clustering 346 simultaneously operating on the rows and columns of a real-valued similarity matrix. This is 347 graphically represented as a 2-dimensional colored image, where each entry of the matrix is 348 colored on the basis of its value, and where the rows and columns are reordered according to 349 the hierarchical clustering. 
In this section, we provide neither a thorough biological interpretation of the results,

353 nor a comprehensive view of every statistical analysis performed. Instead, we highlight the

354 limits of each method leading to the next step of the statistical analysis and show how a biologist can interpret and take over the conclusions of a statistical study.

We illustrate the bivariate analysis through some graphical representations of phenotypic data linked to one parameter of the experimental design. Figure 5A displays parallel boxplots as well as individual observations of the number of leaves for the 5 ecotypes triplicate for each ecotype and temperature. data. The relatively low scattering of points representing individuals of each biological

364 replicate (Figure 5A) indicates a rather good reproducibility between all the samples and between the repetitions. So, the values from several plants of a given biological repetition can

366 be averaged, to go on with the analyses. The visual impression provided by Figure 5B 367 regarding the temperature and ecotype effects can be confirmed via statistical testing such as 368 two-way ANOVA (Bingham \& Fry) (results not shown). However, this kind of analysis does 369 not provide any information about the potential relationships between several variables. This drawback justifies the next step of analysis which deals with a whole data set.

374 set. It is composed of 364 variables (or transcripts). The first way to question the whole data 375 set can be through the computation of pairwise correlation coefficients. For instance, Figure 6 
376 displays the correlation matrix between samples. It indicates that the levels of gene expression

377 for each sample are positively correlated (only green color and identically oriented ellipses)

378 with all the others.

Then, a PCA can be performed as an extension of the quality control. For instance,

380 Figure 7A highlights the distance between the three replicates corresponding to one condition.

381 We can observe that the Grip ecotype is well gathered, whereas the Col ecotype is more

382 scattered. This information must be moderated because of the rather low proportion of

383 variance explained by the first two principal components displayed here. Having a look at the

384 following components could be meaningful to consolidate and complete this information.

However, the interpretation of the PCA brings a first trend. Indeed, the samples are

386 clearly separated along the first (horizontal) axis according to the temperature: samples at

$38722^{\circ} \mathrm{C}$ are all located on the left (negative coordinates on PC1), whereas samples at $15^{\circ} \mathrm{C}$ are

388 on the right. This indicates that the effect of temperature is stronger than that of ecotypes

389 because PC1 capture the most important source of variability in the data. The representation

390 of the variables, i.e. the transcripts (Figure 7B), is not of great interest at this step; it mainly

391 highlights the need for selection methods to facilitate the interpretation of the results in terms

392 of gene expression level. However, the interpretation of such a plot jointly with the individual

393 plot enables, for instance, identifying over-expressed genes in samples at $15^{\circ} \mathrm{C}$ : they are

394 located on the right of the variables plot (in the same area as samples at $15^{\circ} \mathrm{C}$ in the individual

395 plot).

397 5.3. Supervised analysis and variable selection

To illustrate a supervised analysis, we deal with the same data set as before (cell wall

399 transcriptomics for the quantitative block) to discriminate the samples according to the 
400

401

402

403

404

405

406

407

408

409 on the quantitative variables. Thus, the biologist can play with these factors to answer its main

424 biological question and to identify potential future prospects.

temperature (qualitative block) by performing a PLS-DA analysis. A similar analysis could be made with the ecotype, but interpretation would be more complicated with 5 categories instead of 2 for temperature. Moreover, we have already seen that the temperature effect is the strongest for this data set (Figure 7A). Furthermore, to address the problem of interpretability of the results, we also consider the sparse version of PLS-DA to select the most discriminant genes for the temperature effect. The number of variables to select has to be determined by the user. It depends on the way the potential candidates will be validated. For instance, if validation has to be done through new biological experiments, the number of selected variables must not be too large (about 10). But, if the validation consists in querying a biological database, this number can be higher (about hundreds).

Figure 7 also displays the results of PLS-DA (C, D) and S-PLS-DA (E, F). Individuals plots (Figure 7C, E) and variables plots (Figure7D, F) are interpreted in the same way as PCAs. Individuals plots only use two colors corresponding to the two temperatures. For both PLS-DA and S-PLS-DA, the discrimination between the samples is clear-cut (Figure 7C, E). This result confirms the overriding effect of the temperature. In other words, the variability due to the five ecotypes does not impede from detecting the temperature effect. The result of S-PLS-DA indicates that the discrimination can be observed with only a few genes. Indeed, the difference between PLS-DA and S-PLS-DA relies on the number of genes involved in the discrimination process. The list of the most relevant genes displayed in Figure 7F has to be investigated through for instance functional analysis, but these developments are outside the scope of this article.

These examples of sparse methods highlight the specificity of a supervised analysis: it enables studying the impact of the factors of the experimental design (here the temperature) 
Multi-block analyses can address the main purpose of an integrative study by analysing together all the blocks acquired for each sample. As an illustration, we expose the

429 results of a five-block supervised analysis focused on the rosettes, considering phenotypic, 430 cell wall transcriptomics, proteomics and metabolomics as quantitative variables and 431 temperature as the qualitative (or categorical) block.

The statistical relationships between blocks must be defined by the user through a design matrix. This matrix is a square of size [(number of blocks) x (number of blocks)], it is

434 symmetrical and contains values between 0 and 1 . A value close to or equal to 1 (respectively 0) indicates a strong relationship (respectively weak or no relationship) between the blocks to

436 be integrated. Fixing the values in the design matrix is crucial and complex because it requires 437 expressing biological relationships as numerical values (e.g. can we consider that the link 438 between proteomics and transcriptomics data is stronger than the link between proteomics and 439 metabolomics data?). For the sake of simplicity, 0 and 1 values can be used in a binary point 440 of view: blocks are linked or not. In a supervised context, the values also enable balancing the 441 optimisation between, on the one hand the relationships between quantitative blocks and, on 442 the other hand, the discrimination of the outcome. In our example, we considered a design 443 matrix composed of 0 between blocks to favor the discrimination task rather than the 444 relationships between the blocks. A full design matrix (composed of 1) highlights more 445 clearly relationships between blocks, but can lead to misclassified samples. The interpretation of a multi-block supervised analysis requires several graphical 447 outputs. Some of them are presented in Figure 8. Figure 8A allows to check whether the 448 correlation between the first components from each data set has been maximized as specified 449 in the design matrix (Tenenhaus et al., 2014). Globally, correlation values are close to 1 and 
mainly due to the separation of the two categories $\left(22\right.$ vs $15^{\circ} \mathrm{C}$; because of our design, this

451 biological experiments (Chawla et al., 2011). relevance networks between variables. On Figure 9A, each selected variable is a node located

matrix favors discrimination). With a full designed matrix, we get higher correlation values but with less separated groups. Regarding the individual plots (Figure 8B), it appears that the discrimination is better for the transcriptomics and proteomics blocks than for the others. The sample plot (Figure 8B) has also to be interpreted regarding the variable plot (Figure 8C). To make the interpretation easier, we present here the results of the sparse version of the multiblock analysis. Therefore, we can identify variables from each block mainly involved in the discrimination according to the temperature. For instance, variables located on the right on the correlation circle plot (Figure 8C) contribute to the discrimination between the samples growing at $22^{\circ} \mathrm{C}$ because they are also located on the right in the individuals plots (Figure 8B). Another way to display the results is presented in Figure 8D. The clustered image map highlights the profiles of selected variables among the samples. It also includes the results of hierarchical clustering performed jointly on variables and samples. Regarding the samples, the two groups based on temperature are visualized through the dendrogram on the left. However, let us note that the cluster gathering the samples at $15^{\circ} \mathrm{C}$ can be split into two sub-clusters with the Col ecotype isolated. Regarding the variables, it mainly points out global trends of the behavior of selected variables. The interpretation can then lead to retro analyses to validate potential candidates. This can be done through new statistical analyses as well as new

\subsection{Relevance networks}

Another way to interpret the results of a multi-block approach consists in producing on a circle. Variables are sorted first according to their block, then depending on their 
474 importance in discrimination. An edge links two nodes if their correlation is higher than a

475 threshold subjectively set by the user (we chose 0.9 in Figure 9A).

The correlations are mainly positive and concern a few variables from each block. To

477 complete the interpretation, we focus on another network generated with only two blocks

478 (Figure 9B, cell wall transcriptomics and proteomics). It accentuates the relationships between

479 pairs of proteins and transcripts. The selection of variables is a precious information for the

480 biologist to focus on some of them for validation and draw conclusions in biological terms.

Relevance networks can also be viewed as a first step to modelling as it mimics

482 biological networks and provides clues to address inference networks issues through further

483 dedicated experiments.

\section{CONCLUSION}

In an integrative biology context, the huge quantity of data produced, which can also

487 be heterogeneous, requires adapted and specific statistical methods tentatively summarized in

488 Figure 2. Even if the multi-block approaches can be viewed as the best tool to address a given

489 issue, other more basic standard statistical methods (univariate for instance) must not be

490 omitted. A deep understanding of a biological phenomenon requires a sequence of various

491 approaches to analyse the data. Finally, we consider that each method contributes to a better

492 interpretation of the others as we intended to express it with the schematic view of the

493 protocol as intertwined cycles (Figure 4). The statistical analysis of the large omics data sets

494 can be a never-ending story because each step of the framework provides information. The

495 results presented in this case study could not have been obtained using standard statistical

496 approaches. Actually, it is our global integrative strategy that led us to novel biological 497 results. 


\section{Acknowledgements}

500 The authors are thankful to the Paul Sabatier-Toulouse 3 University and to the Centre

501 National de la Recherche Scientifique (CNRS) for granting their work. This work was also

502 supported by the French Laboratory of Excellence project "TULIP" (ANR-10-LABX-41;

503 ANR-11-IDEX-0002-02). HD was supported by the Midi-Pyrénées Region and the Federal

504 University of Toulouse. Thanks to Dr Kim-Anh Lê Cao, Pr Philippe Besse and François

505 Bartolo for their support and help with the graphical outputs and interpretation of the multi-

506 block analysis.

508 Supplementary Files:

$509 \quad$ Supplementary Table S1. Toy data set containing 12 observations and 3 variables. 


\section{Figure captions:}

512 Figure 1. Scatterplot representing Vy values (vertical axis) according to Vx values (horizontal

513 axis). Control and treated observations are represented with grey triangles and black circles,

514 respectively.

515 Figure 2. Workflow for our multi-omics integrative studies. The different parts of this article

516 are represented with grey boxes and the green boxes close the workflow with biological 517 concepts. The workflow converges towards the functional analysis required to validate the 518 whole study.

519 Figure 3. Schematic overview of the strategy and experimental protocol used in this study. 520 Each circle represents one plant and each color stands for one ecotype of A. thaliana. For each 521 of the three biological replicates, the position of a given ecotype has been changed randomly 522 to avoid position effects.

523 Figure 4. One purpose, one method to analyse qualitative and quantitative blocks. A)

524 Schematic representation of the different blocks (or data sets) co-analysed in this study. The 525 samples are represented in rows and the variables in columns. B) Schematic overview of the 526 methods implemented represented by cycles within an integrative study. C) Examples of 527 graphical outputs detailed in the results section. PCA: Principal Component Analysis; MB: 528 Multi-Blocs; PLS: Partial Least Squares regression; DA: Discriminant Analysis. Qualitative 529 and quantitative blocks are represented in green and grey respectively.

530 Figure 5. Examples of graphical outputs of a supervised bivariate analysis illustrated by A) A 531 boxplot (each color corresponds to the different values obtained for each triplicate) and B) An 532 individual plot. (each color corresponds to the average obtained for one triplicate, and does 533 not match with color used in A). The number of leaves for 5 ecotypes of A. thaliana (Col, 534 Roch, Grip, Hern and Hosp) and 2 growth temperatures (22 and $\left.15^{\circ} \mathrm{C}\right)$ was used. These plots 
535 were obtained using functions geom_point() and geom_boxplot() from the ggplot2 package 536 (Wickham, 2016).

537 Figure 6. A graphical representation of the multivariate analysis, pairwise correlation 538 coefficients of cell wall transcriptomics data sets in the rosettes of the five A. thaliana 539 ecotypes grown at $15^{\circ} \mathrm{C}$ or $22^{\circ} \mathrm{C}$. The color code and the ellipse size represent the correlation 540 coefficient between the levels of expression of genes for each sample. The areas and the 541 orientations of the ellipses represent the absolute value of the corresponding correlation 542 coefficients. The eccentricity of the ellipses represents the absolute value of the corresponding 543 correlation coefficients. This plot was obtained using the function corrplot() from the corrplot 544 package (Wei \& Simko, 2016).

545 Figure 7. Graphical representation of the unsupervised (A, B) and supervised (C-F) analysis 546 of the rosette cell wall transcriptomes from ecotypes grown at $22^{\circ} \mathrm{C}$ and $15^{\circ} \mathrm{C}$. A) Individuals 547 plot of a PCA from ecotypes grown at $22^{\circ} \mathrm{C}$ (bright color) and $15^{\circ} \mathrm{C}$ (pale color) associated to 548 the B) Variables plot. C) Individuals plot of a PLS-DA from ecotypes grown at $22^{\circ} \mathrm{C}$ (orange) 549 and $15^{\circ} \mathrm{C}$ (blue) associated to the D) Variables plot and E) Individuals plot of a S-PLS-DA 550 associated to the E) Variables plot. Two circles of radius 1 and 0.5 are plotted in each 551 variables plot to reveal the correlation structure of the variables. These plots were obtained 552 using the functions pca(), plsda(), plotIndiv() and plotVar() from the mixOmics package 553 (Rohart et al., 2017).

554 Figure 8. A graphical representation of a multi-block analysis realised on the rosettes of ecotypes grown at $22^{\circ} \mathrm{C}$ (orange) and $15^{\circ} \mathrm{C}$ (blue). A) plotDIABLO shows the correlation

556 between components from each data set maximized as specified in the design matrix. B)

557 Individuals plot projects each sample into the space spanned by the components of each block 558 associated to the C) Variables plot that highlights the contribution of each selected variable to 559 each component, D) Clustered image map of the variables (Protein: red; Transcripts: green; 
560 Metabolites: grey; Phenotypes: black) to represent the multi-omics profiles for each sample

$561\left(15^{\circ} \mathrm{C}\right.$ : blue, $22^{\circ} \mathrm{C}$ : orange). These plots were obtained using the functions block.splsda(),

562 plotIndiv(), plotVar() and $\operatorname{cim}()$ from the mixOmics package (Rohart et al., 2017).

563 Figure 9. Example of network representation. A) A Circos plot represents the correlations

564 between variables within and between each block (edges inside the circle) and shows the

565 average value of each variable in each condition (line profile outside the circle). B) A network

566 displaying the correlation between the transcriptomics (.T, green) and the proteomics data (.P,

567 red) colored from blue to red according to the color key. These plots were obtained using the

568 functions circosPlot() and network() from the package mixOmics (Rohart et al., 2017). 


\section{References}

571 Anscombe, F. J. (1973). Graphs in statistical analysis. Am. Stat. 27(1), 17-21.

572 Argelaguet, R., Velten, B., Arnol, D., Dietrich, S., Zenz, T., Marioni, J.C., Buettner, F., Huber, W. \& Stegle, O. (2018). Multi Omics Factor Analysis-a framework for unsupervised integration of multi omics data sets. Mol. Syst. Biol. 14:e8124 https:// doi.org/10.15252/msb.20178124

576

577

578

579

580

581

Bingham, N. H., \& Fry, J. M. (2010). Regression: Linear models in statistics: Springer Science \& Business Media.

Bécue-Bertaut, M., \& Pagès, J. (2008). Multiple factor analysis and clustering of a mixture of quantitative, categorical and frequency data. Comput. Stat. Data Anal, 52(6), 32553268.

Carey, M. A., \& Papin, J. A. (2018). Ten simple rules for biologists learning to program. PLoS Comput Biol, 14(1), e1005871. doi:10.1371/journal.pcbi.1005871

Chawla, K., Barah, P., Kuiper, M., \& Bones, A. M. (2011). Systems biology: a promising tool to study abiotic stress responses. Omics and Plant Abiotic Stress Tolerance, 163-172. doi:10.2174/97816080505811110101

Cumming, G., Fidler, F., \& Vaux, D. L. (2007). Error bars in experimental biology. J. Cell. Biol., 177(1), 7-11. doi:10.1083/jcb.200611141

Duruflé, H., Hervé, V., Ranocha, P., Balliau, T., Zivy, M., Chourré, J., San Clemente, H., Burlat, V., Albenne, C., Déjean, S., Jamet, E., Dunand, C. (2017). Cell wall modifications of two Arabidopsis thaliana ecotypes, Col and Sha, in response to suboptimal growth conditions: an integrative study. Plant Sci. 263, 183-193. doi: 10.1016/j.plantsci.2017.07. 015 
594 Duruflé, H., Albenne, C., Jamet, E. \& Dunand, C. Phenotyping and cell wall polysaccharide 595 composition of five Arabidopsis ecotypes grown at optimal or sub-optimal temperatures, Data in brief (2019) (in press)

597

600

601

602

603

604

605

606

607

608

609

610

611

612

613

614

615

616

617

618 proteomic datasets of rosettes and floral stems from five Arabidopsis thaliana ecotypes grown at optimal or suboptimal temperature, Data in brief (2019) (in revision)

Friendly, M. (2002). Corrgrams: Exploratory displays for correlation matrices. Am. Stat., 56(4), 316-324.

Gentleman, R. C., Carey, V. J., Bates, D. M., Bolstad, B., Dettling, M., Dudoit, S., . . Zhang, J. (2004). Bioconductor: open software development for computational biology and bioinformatics. Genome Biol., 5(10), R80. doi:10.1186/gb-2004-5-10-r80

González, I., Lê Cao, K. A., Davis, M., \& Déjean, S. (2013). Insightful graphical outputs to explore relationships between two ‘omics’ data sets. BioData Min., 5, 19.

Gray, S. B., \& Brady, S. M. (2016). Plant developmental responses to climate change. Dev. Biol., 419(1), 64-77. doi:10.1016/j.ydbio.2016.07.023

Günther, O. P., Shin, H., Ng, R. T., McMaster, W. R., McManus, B. M., Keown, P. A., . . Lê Cao, K. A. (2014). Novel multivariate methods for integration of genomics and proteomics data: applications in a kidney transplant rejection study. OMICS, 18(11), 682-695.

Hoffmann, M., H. (2002). Biogeography of Arabidopsis thaliana (L.) Heynh. (Brassicaceae). J. Biogeogr., 29(1), 125--134. doi:10.1046/j.1365-2699.2002.00647.x

Houben, K., Jolie, R., Fraeye, I., Van Loey, A., \& Hendrickx, M. (2011). Comparative study of the cell wall composition of broccoli, carrot, and tomato: Structural characterization of the extractable pectins and hemicelluloses. Carbohydr. Res., 346(9), 1105-1111. doi:10.1016/j.carres.2011.04.014 
619 Husson, F., \& Josse, J. (2013). Handling missing values with/in multivariate data analysis 620 (principal component methods). Agrocampus Ouest-Laboratoire de mathématique appliquée, Rennes.

622 Jamet, E., Roujol, D., San Clemente, H., Irshad, M., Soubigou-Taconnat, L., Renou, J. P., \& Pont-Lezica, R. (2009). Cell wall biogenesis of Arabidopsis thaliana elongating cells: transcriptomics complements proteomics. BMC Genomics, 10, 505. doi:10.1186/14712164-10-505

626

627

628

629

630

631

632

Kerr, M. K. (2003). Experimental design to make the most of microarray studies. Methods Mol. Biol., 224, 137-147. doi:10.1385/1-59259-364-X:137

Li, Y., Wu, F. X., \& Ngom, A. (2016). A review on machine learning principles for multiview biological data integration. Brief Bioinform. doi:10.1093/bib/bbw113

Lê Cao, K. A., Boitard, S., \& Besse, P. (2011). Sparse PLS discriminant analysis: biologically relevant feature selection and graphical displays for multiclass problems. BMC Bioinformatics, 12, 253. doi:10.1186/1471-2105-12-253

Lê Cao, K. A., González, I., \& Déjean, S. (2009). integrOmics: an R package to unravel relationships between two omics datasets. Bioinformatics, 25(21), 2855-2856. doi:10.1093/bioinformatics/btp515

Maier, T., Güell, M., \& Serrano, L. (2009). Correlation of mRNA and protein in complex biological samples. FEBS Lett, 583(24), 3966-3973. doi:10.1016/j.febslet.2009.10.036

Mardia, K. V., Kent, J. T., \& Bibby, J. M. (1980). Multivariate analysis (probability and mathematical statistics). Academic Press London.

Matejka, J., \& Fitzmaurice, G. (2017). Same stats, different graphs: Generating datasets with varied appearance and identical statistics through simulated annealing. Paper presented at the Proceedings of the 2017 CHI Conference on Human Factors in Computing Systems. 
McDonald, J. H. (2009). Handbook of biological statistics (Vol. 2): Sparky House Publishing Baltimore, MD.

Meng, C., Zeleznik, O. A., Thallinger, G. G., Kuster, B., Gholami, A. M., \& Culhane, A. C. (2016). Dimension reduction techniques for the integrative analysis of multi-omics data. Brief Bioinform., 17(4), 628-641. doi:10.1093/bib/bbv108

Murdoch, D. J., \& Chow, E. D. (1996). A graphical display of large correlation matrices. The Am. Stat., 50(2), 178-180.

R Core Team, (2018). R: A Language and Environment for Statistical Computing.

Rai, A., Saito, K., \& Yamazaki, M. (2017). Integrated omics analysis of specialized metabolism in medicinal plants. Plant J., 90(4), 764-787. doi:10.1111/tpj.13485

Rajasundaram, D. Selbig, J. (2016). More effort - more results: recent advances in integrative 'omics' data analysis, Curr. Opin. Plant Biol., 30, 57-61, https://doi.org/ 10.1016/j.pbi.2015.12.010.

Rohart, F., Gautier, B., Singh, A., \& Lê Cao, K. A. (2017). mixOmics: An R package for 'omics feature selection and multiple data integration. PLoS Comput. Biol., 13(11), e1005752. doi:10.1371/journal.pcbi.1005752

Sabatier, R., Vivien, M., \& Reynès, C. (2013). Une nouvelle proposition, l'analyse discriminante multitableaux: Statis-lda. Journal de la Société Française de Statistique, 154(3), 31-43.

Savo, V., Lepofsky, D., Benner, J. P., Kohfeld, K. E., Bailey, J., \& Lertzman, K. (2016). Observations of climate change among subsistence-oriented communities around the world. Nat. Clim. Change, 6(5), 462-473.

Sibout, R. (2017). Crop breeding: Turning a lawn into a field. Nat Plants, 3, 17060. doi:10.1038/nplants.2017.60 
668 Singh, A., Gautier, B., Shannon, C. P., Vacher, M., Rohart, F., Tebutt, S. J., \& Le Cao, K. A. 669 (2019). DIABLO-an integrative, multi-omics, multivariate method for multi-group classification. Bioinformatics, doi:10.1093/bioinformatics/bty1054.

671 Tenenhaus, A., Philippe, C., Guillemot, V., Lê Cao, K. A., Grill, J., \& Frouin, V. (2014). Variable selection for generalized canonical correlation analysis. Biostatistics, 15(3), 569-583. doi:10.1093/biostatistics/kxu001

674 Tibshirani, R. (1996). Regression shrinkage and selection via the lasso. J. R. Stat. Soc. Ser. B-Stat. Methodol., 267-288.

676 Voillet, V., Besse, P., Liaubet, L., San Cristobal, M., \& González, I. (2016). Handling missing rows in multi-omics data integration: multiple imputation in multiple factor analysis framework. BMC Bioinformatics, 17(1), 402. doi:10.1186/s12859-016-1273-5

679 Wei, T., \& Simko, V. (2016). corrplot: Visualization of a Correlation Matrix. R package version 0.77. CRAN, Vienna, Austria.

681 Wickham, H. (2014). Tidy data. Journal of Statistical Software, 59(10), 1-23.

682 Wickham, H. (2016). ggplot2: elegant graphics for data analysis: Springer.

683 Wold, S., Sjöström, M., \& Eriksson, L. (2001). PLS-regression: a basic tool of chemometrics. Chemometrics Intell. Lab. Syst., 58(2), 109-130.

685 Zargar, S. M., Gupta, N., Nazir, M., Mir, R. A., Gupta, S. K., Agrawal, G. K., \& Rakwal, R. 686 (2016). Omics-A New Approach to Sustainable Production. In Breeding Oilseed Crops for Sustainable Production (pp. 317-344): Elsevier. 


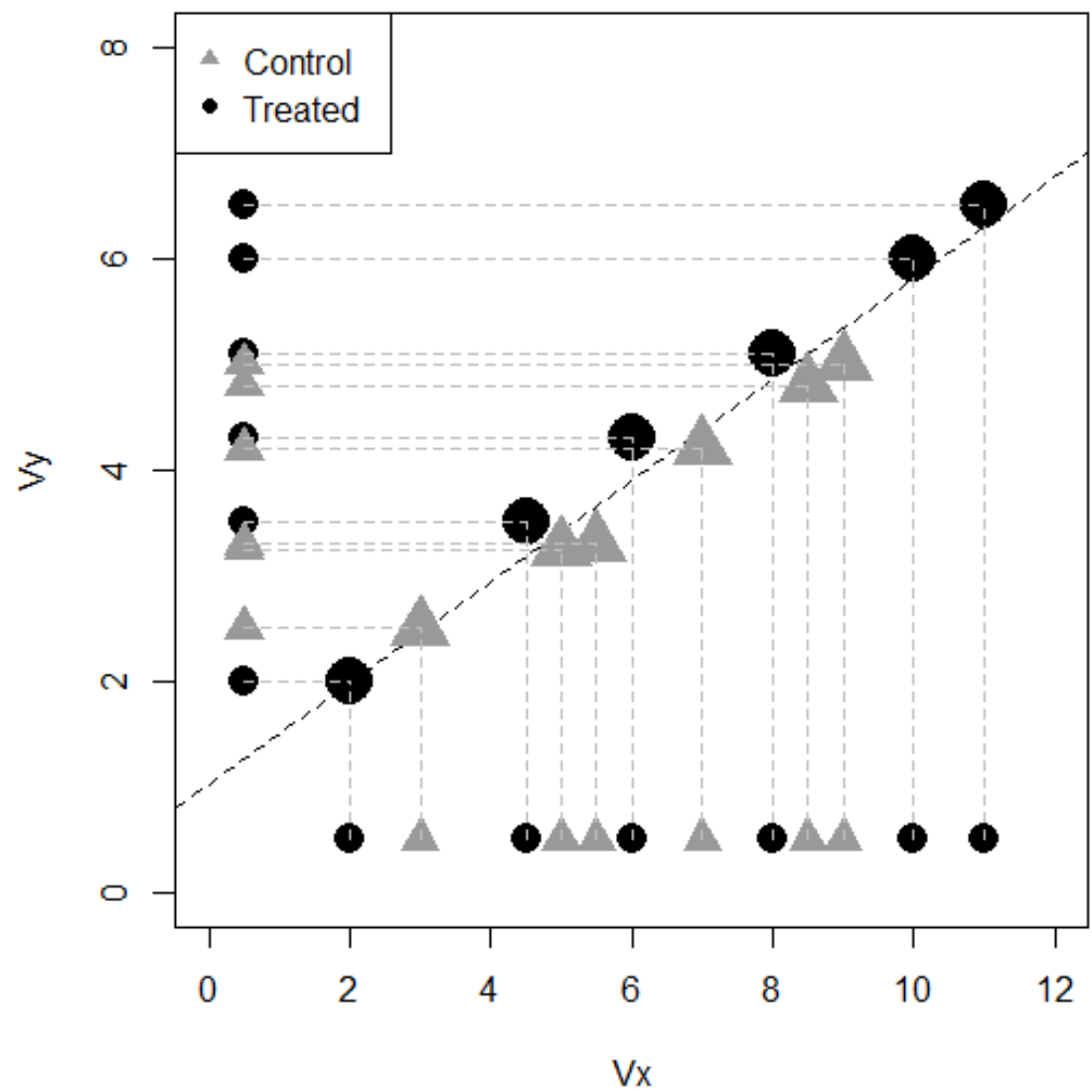

Figure 1. Scatterplot representing Vy values (vertical axis) according to Vx values (horizontal axis). Control and treated observations are represented with grey triangles and black circles, respectively. 


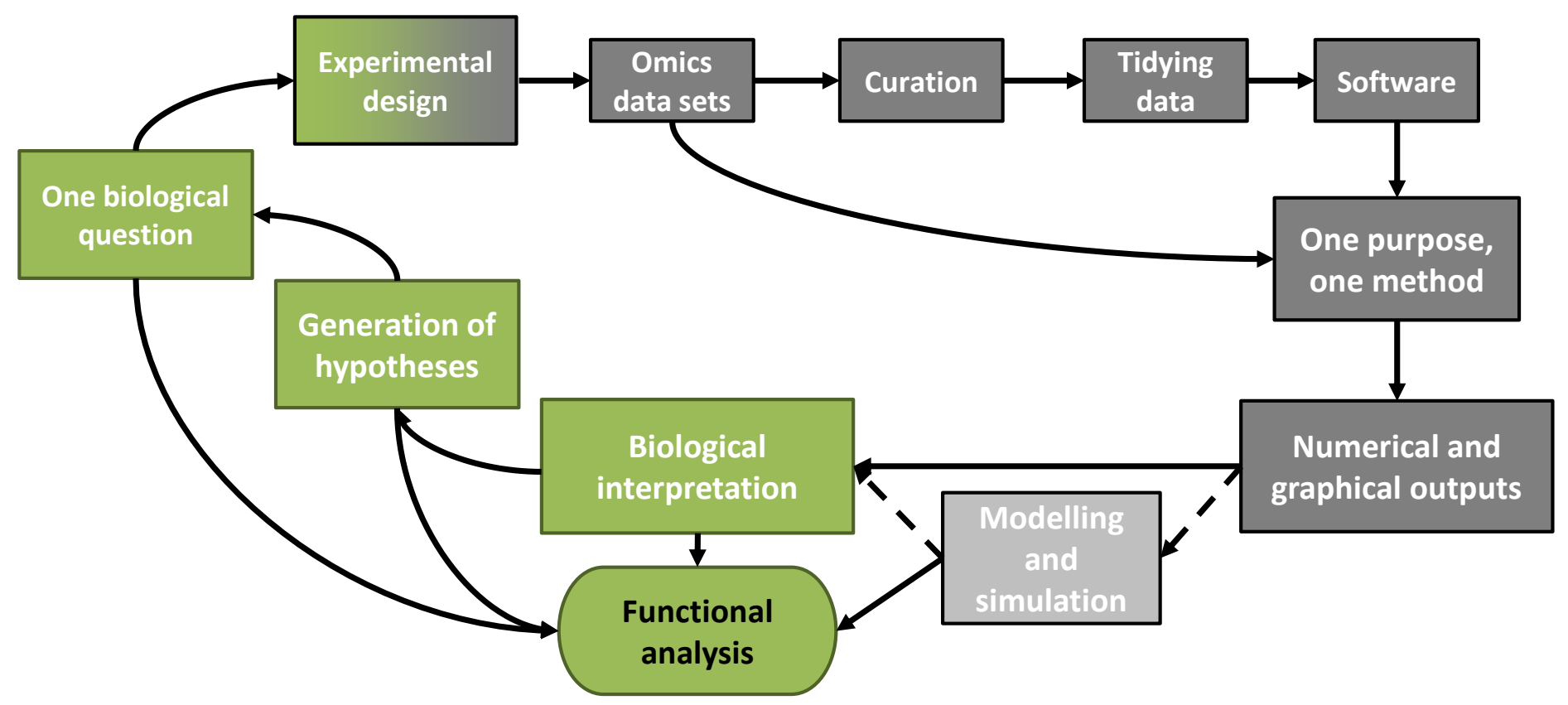

Figure 2. Workflow for our multi-omics integrative studies. The different parts of this article are represented with grey boxes and the green boxes close the workflow with biological concepts. The workflow converges towards the functional analysis required to validate the whole study. 

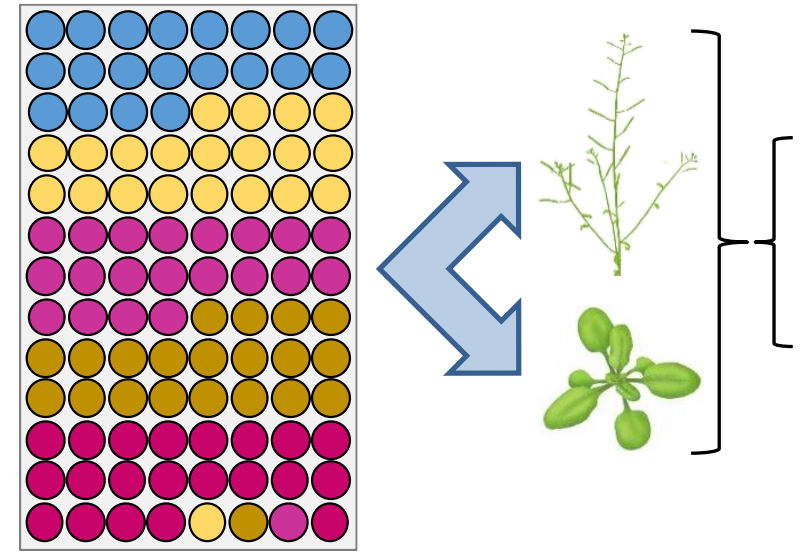

\section{$\rightarrow$ Phenomics}

$\rightarrow$ Metabolomics

$\rightarrow$ Proteomics

$\rightarrow$ Transcriptomics

Figure 3. Schematic overview of the strategy and experimental protocol used in this study. Each circle represents one plant and each color stands for one ecotype of $A$. thaliana. For each of the three biological replicates, the position of a given ecotype has been changed randomly to avoid position effects. 
bioRxiv preprint doi: https://doi.org/10.1101/357921; this version posted July 25, 2019. The copyright holder for this preprint (which was

A
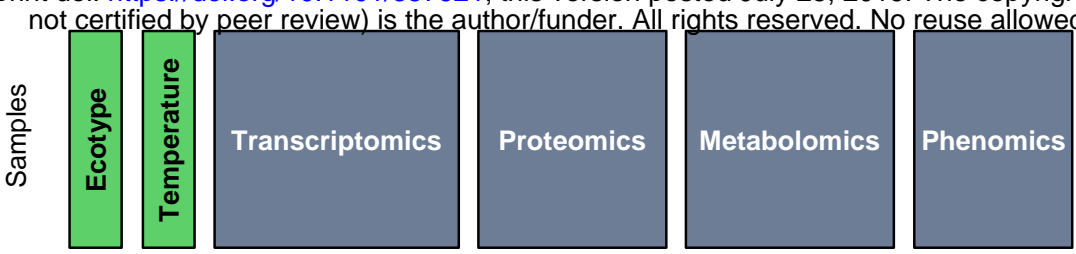

B

Data

Cycles

C

Graphical outputs

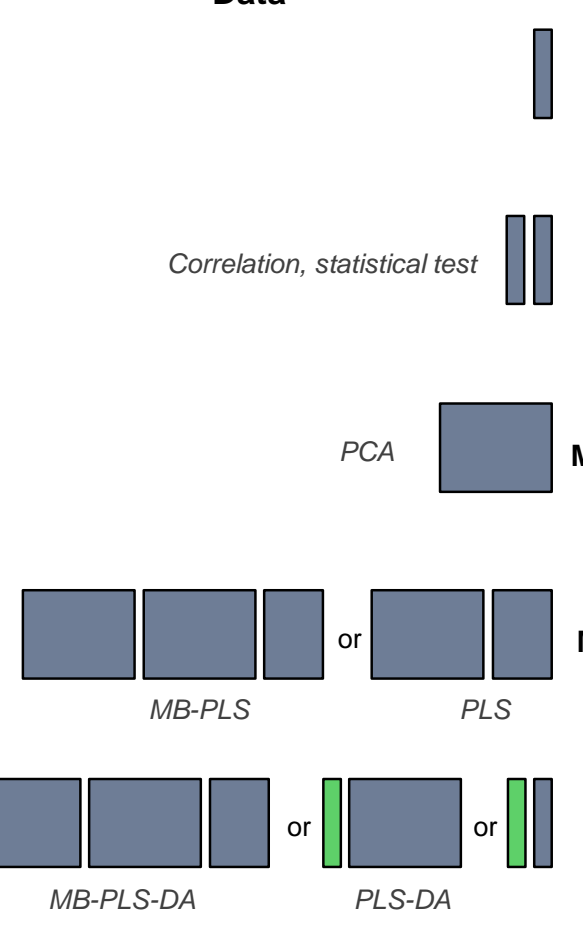

Supervise

d

Fig. 1 and 5

Fig. 6

Fig. 7 A, $B$

Fig. $7 C, D$

Fig. 8 and 9

Figure 4. One purpose, one method to analyse qualitative and quantitative blocks. A) Schematic representation of the different blocks (or data sets) co-analysed in this study. The samples are represented in rows and the variables in columns. B) Schematic overview of the methods implemented represented by cycles within an integrative study. C) Examples of graphical outputs detailed in the results section. PCA: Principal Component Analysis; MB: Multi-Blocs; PLS: Partial Least Squares regression; DA: Discriminant Analysis. Qualitative and quantitative blocks are represented in green and grey respectively. 
A bioRxiv preprint doi: https://doi.org/10.1101/357921; this version posted July 25,2019 . The copyyght holder for this preprint (which was
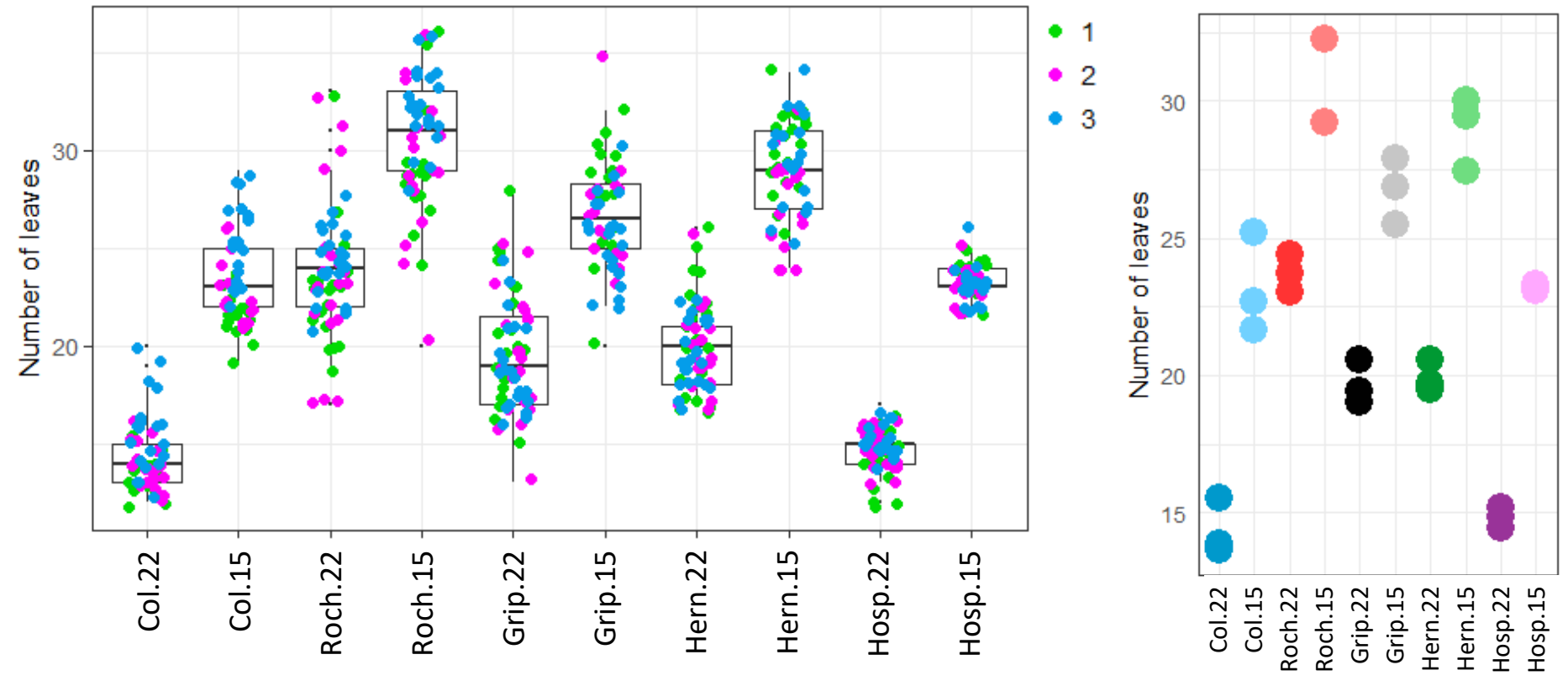

Figure 5. Examples of graphical outputs of a supervised bivariate analysis illustrated by A) A boxplot (each color corresponds to the different values obtained for each triplicate) and B) An individual plot. (each color corresponds to the average obtained for one triplicate, and does not match with color used in A). The number of leaves for 5 ecotypes of $A$. thaliana (Col, Roch, Grip, Hern and Hosp) and 2 growth temperatures ( 22 and $15^{\circ} \mathrm{C}$ ) was used. These plots were obtained using functions geom_point() and geom_boxplot() from the ggplot2 package (Wickham, 2016). 


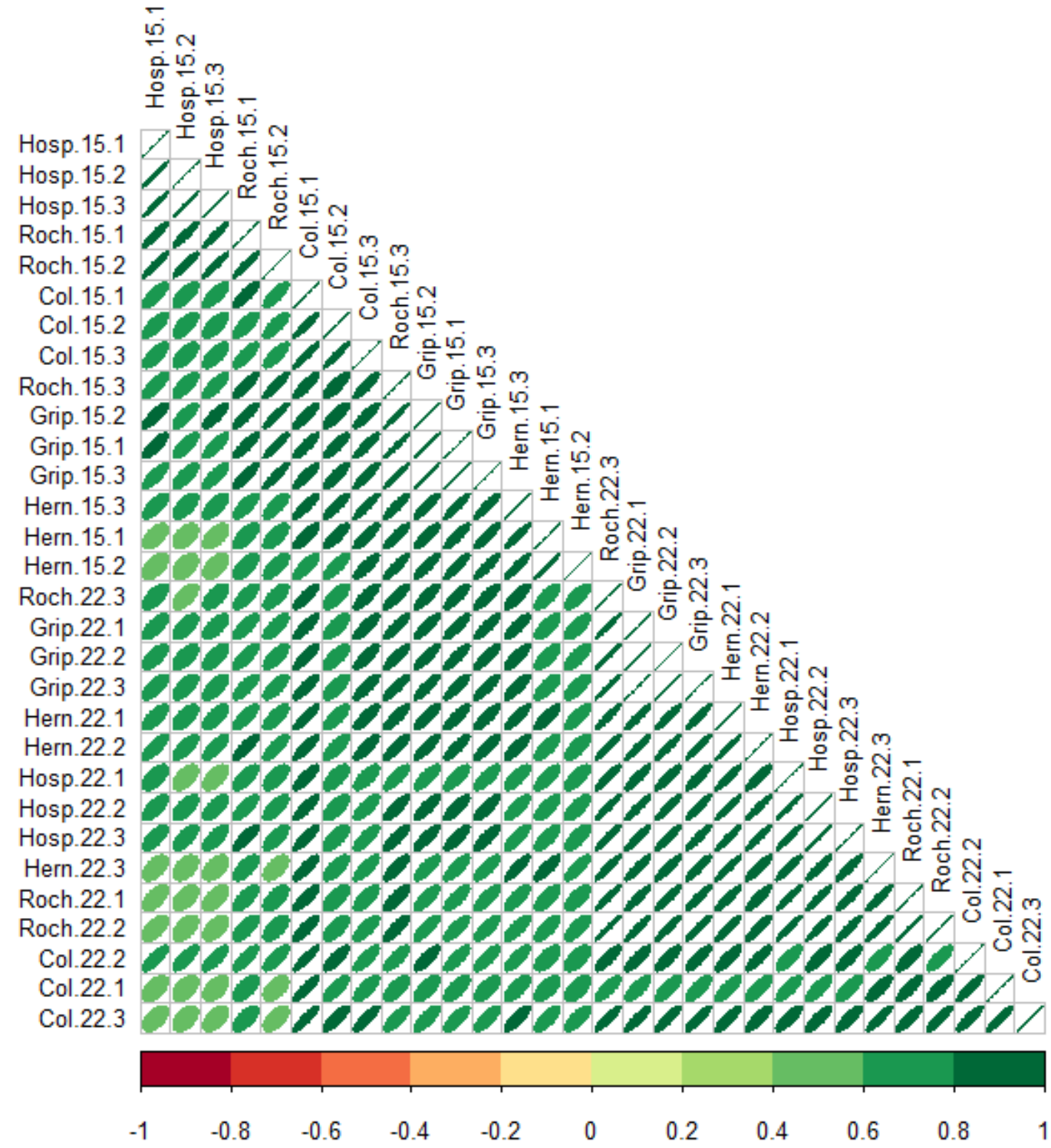

Figure 6. A graphical representation of the multivariate analysis, pairwise correlation coefficients of cell wall transcriptomics data sets in the rosettes of the five $A$. thaliana ecotypes grown at $15^{\circ} \mathrm{C}$ or $22^{\circ} \mathrm{C}$. The color code and the ellipse size represent the correlation coefficient between the levels of expression of genes for each sample. The areas and the orientations of the ellipses represent the absolute value of the corresponding correlation coefficients. The eccentricity of the ellipses represents the absolute value of the corresponding correlation coefficients. This plot was obtained using the function corrplot() from the corrplot package (Wei \& Simko, 2016). 
bioRxiv preprint doi: https://doi.org/10.1101/357921; this version posted July 25, 2019. The copyright holder for this preprint (which was

A

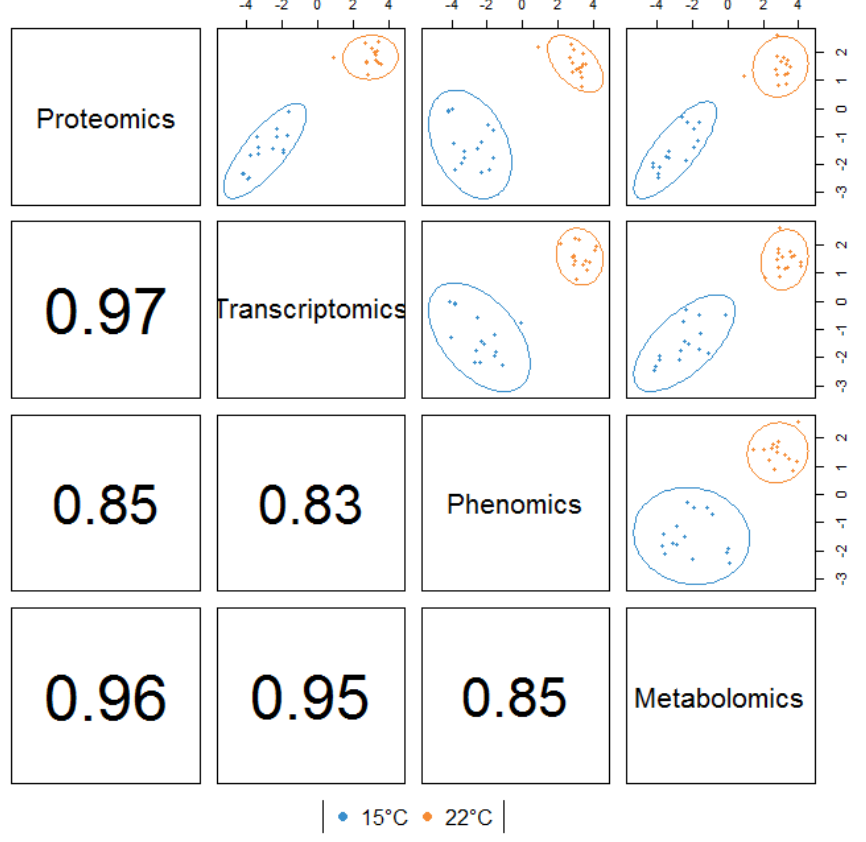

C

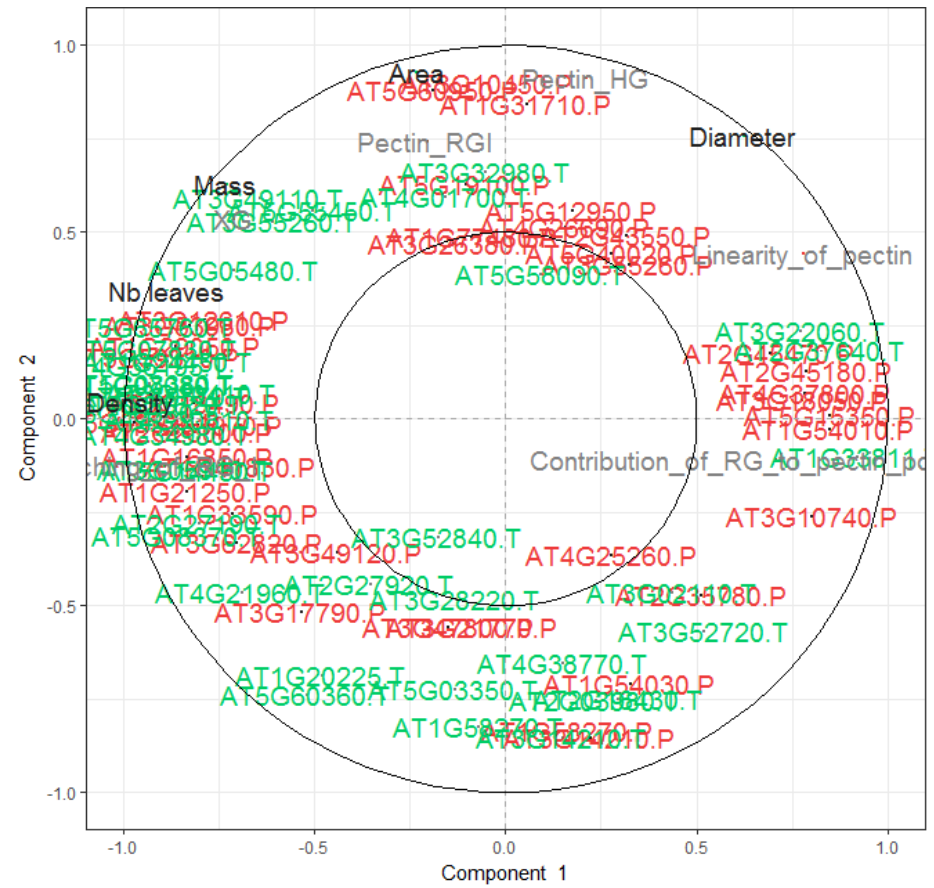

$\mathrm{B}$
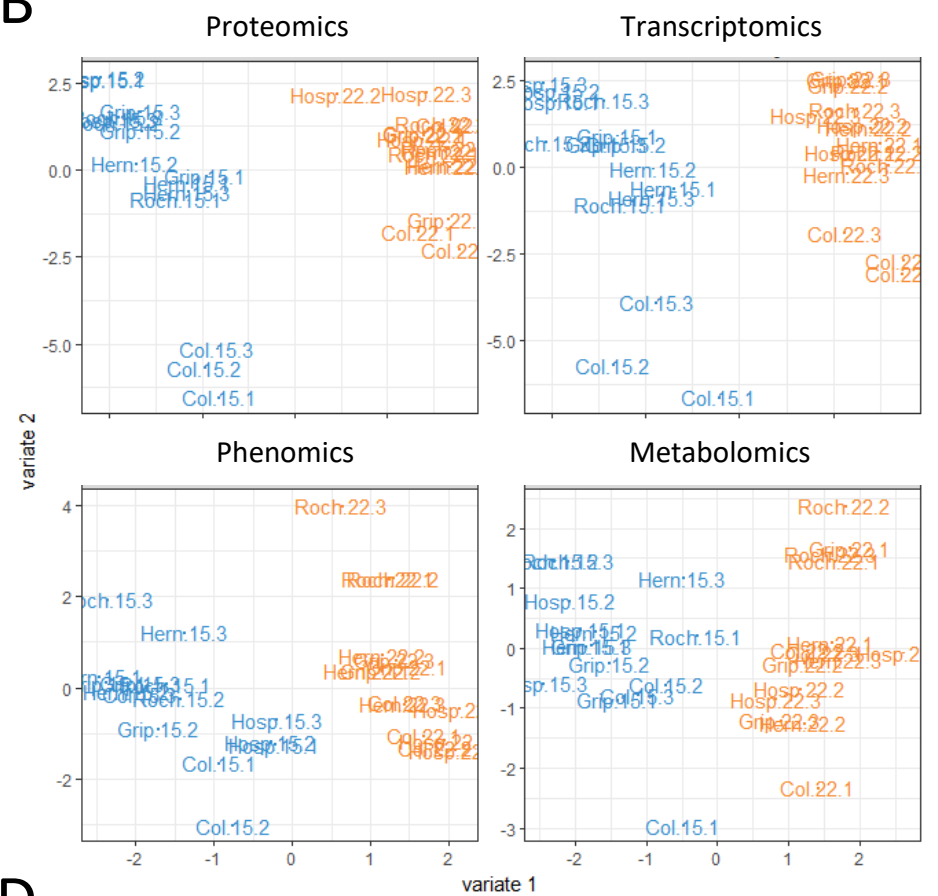

D

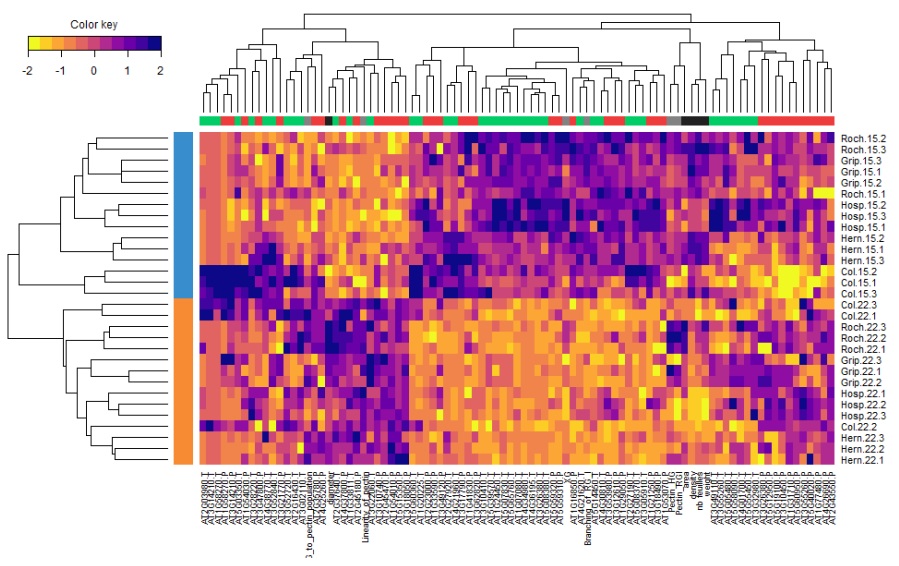

Figure 8. A graphical representation of a multi-block analysis realised on the rosettes of ecotypes grown at $22^{\circ} \mathrm{C}$ (orange) and $15^{\circ} \mathrm{C}$ (blue). A) plotDIABLO shows the correlation between components from each data set maximized as specified in the design matrix. B) Individuals plot projects each sample into the space spanned by the components of each block associated to the C) Variables plot that highlights the contribution of each selected variable to each component, D) Clustered image map of the variables (Protein: red; Transcripts: green; Metabolites: grey; Phenotypes: black) to represent the multi-omics profiles for each sample $\left(15^{\circ} \mathrm{C}\right.$ : blue, $22^{\circ} \mathrm{C}$ : orange). These plots were obtained using the functions block.splsda(), plotIndiv(), plotVar() and cim() from the mixOmics package (Rohart et al., 2017). 
A

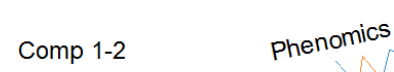

B

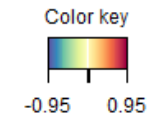

$$
r=0.9
$$

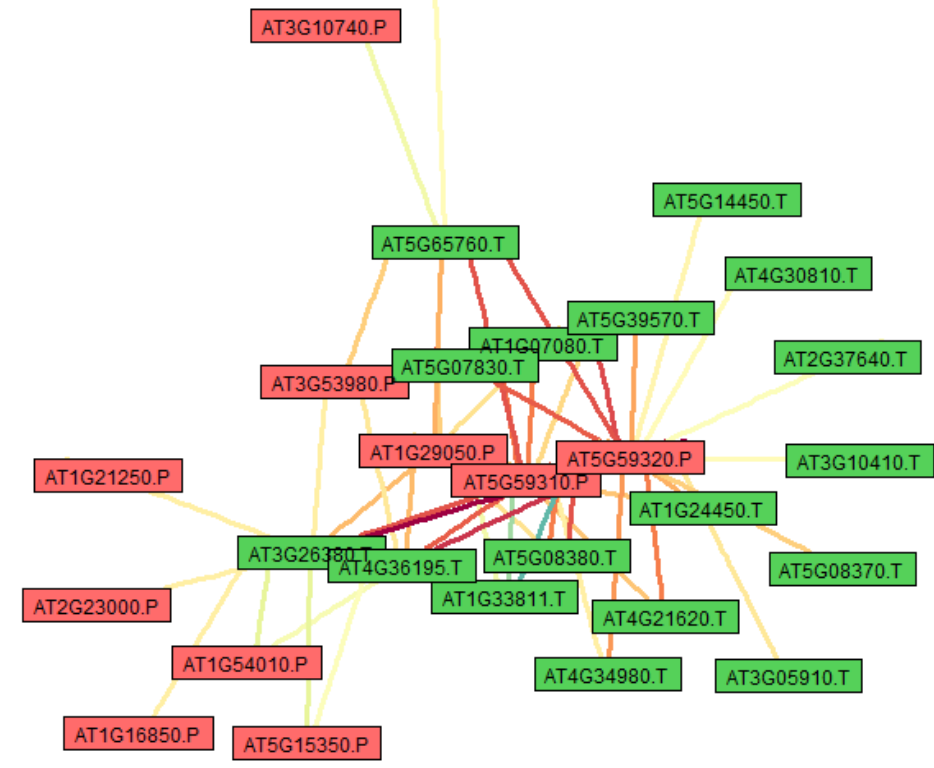

- Positive Correlation

Conditions Expression

$15^{\circ} \mathrm{C}$
$-22^{\circ} \mathrm{C}$

Figure 9. Figure 9. Example of network representation. A) A Circos plot represents the correlations between variables within and between each block (edges inside the circle) and shows the average value of each variable in each condition (line profile outside the circle). B) A network displaying the correlation between the transcriptomics (.T, green) and the proteomics data (.P, red) colored from blue to red according to the color key. These plots were obtained using the functions circosPlot() and network() from the package mixOmics (Rohart et al., 2017). 